Revue d'histoire de l'Amérique française

ARS REVUE D.HISTOIRE DE L'AMÉRIQUE FRANÇAISE

\title{
Le voyage de Samuel Champlain aux Indes occidentales
}

\section{L.-A. Vigneras}

Volume 11, numéro 2, septembre 1957

URI : https://id.erudit.org/iderudit/301831ar

DOI : https://doi.org/10.7202/301831ar

Aller au sommaire du numéro

Éditeur(s)

Institut d'histoire de l'Amérique française

ISSN

0035-2357 (imprimé)

1492-1383 (numérique)

Découvrir la revue

Citer cet article

Vigneras, L.-A. (1957). Le voyage de Samuel Champlain aux Indes occidentales. Revue d'histoire de l'Amérique française, 11(2), 163-200.

https://doi.org/10.7202/301831ar d'utilisation que vous pouvez consulter en ligne.

https://apropos.erudit.org/fr/usagers/politique-dutilisation/ 


\section{LE VOYAGE DE SAMUEL CHAMPLAIN AUX INDES OCCIDENTALES *}

\section{INTRODUCTION}

Lorsque le Brief Discours des choses plus remarquables que Samuel Champlain de Brouage a reconnues aux Indes Occidentales fut publié pour la première fois en 1859 par la Hakluyt Society, en traduction anglaise basée sur l'unique manuscrit alors connu, les éditeurs ne mirent point en doute son authenticité ni sa véracité. Plus tard parurent deux éditions du texte original français, basées également sur le manuscrit de Providence. ${ }^{1}$ Leurs éditeurs y avaient relevé certaines incongruités, mais ils n'y attachèrent aucune importance, puisque la crédulité de Champlain et sa négligence en matière de chronologie étaient connues des historiens. Morris Bishop a été le premier, en 1948, à faire certaines réserves sur la sincérité de l'auteur du Brief Discours; ${ }^{2}$ et deux ans plus tard Jean Bruchesi a publié un article intitulé Champlain a-t-il menti ? ${ }^{3}$ Mais ni Bishop, ni Bruchesi n'ont mis en doute l'authenticité du manuscrit de Providence, base des trois éditions. Ils admettaient à la rigueur que le texte du manuscrit puisse être le travail d'un copiste, mais ils ne doutaient pas que les dessins et les cartes ne fussent l'œuvre de Champlain.

On connaît maintenant l'existence de trois manuscrits du Brief Discours, celui de Providence, déjà nommé, et ceux de Turin (Archivio di Stato) et de Bologne (Biblioteca Universitaria).4 Le texte de Turin se trouve dans un manuscrit qui

* Cet article a déjà paru, mais seulement pour une part, dans l'Annuario de Estudios Americanos, (vol. X, 1953). Avec l'autorisation de l'Annuario, nous en publions ici une version française préparée par l'auteur.

(La Direction)

1 La première est de l'abbé Laverdière (Québec, 1870); la seconde est de la Champlain Society (Toronto, 1922). Avant son acquisition par la John Carter Brown Library, le Manuscrit de Providence se trouvait dans une bibliothèque privée à Dieppe (France) .

2 Morris Bishop, Champlain, A Life of Fortitude (Knopf, N.Y., 1948).

3 Cahier des Dix, numéro 15 (Montréal, 1950).

4 Une analyse du contenu de ces manuscrits se trouve dans Paolo Revelli, Terre d'America e archivi Italiani (Milano, 1926). 
contient aussi le récit d'une expédition française dans le nord du Brésil vers la fin du $16^{\mathrm{e}}$ siècle, lequel s'intitule La Navigation de françois au pays de topinamboux et margaias. La version turinoise du Brief Discours est sans aucun doute l'œuvre d'un compilateur et par conséquent dépourvue de tout intérêt.

Restent les manuscrits de Bologne et de Providence. Une étude comparée révèle que ni l'un ni l'autre n'est l'original qui a dû se perdre ou reste encore à découvrir. Ces deux manuscrits remontent tous les deux au début du $17^{\mathrm{e}}$ siècle. La seule différence importante se trouve dans les habitudes orthographiques et la forme des lettres; le texte lui-même varie fort peu. Cependant, par l'orthographe des noms propres, le manuscrit de Bologne paraît se rapprocher davantage du texte original. Par exemple, dans le manuscrit de Providence, Pedro de Zubiaur apparaît trois fois sous la forme "Soubriago» tandis que le scribe de Bologne, après avoir écrit «Soubriago » les deux premières fois, nomme ensuite correctement le général espagnol sous la forme de «Subiaure ». De même, l'île Caymitos est correctement désignée, sur la carte de La Yaguana, dans le manuscrit de Bologne, par contre dans celui de Providence nous trouvons la forme "Cayima». Sur le croquis de l'embouchure du Guadalquivir, le port de Rota est écrit «Rotes » dans le manuscrit de Bologne, et « Roses » dans l'autre.

Les deux manuscrits sont illustrés. Celui de Providence contient 62 cartes et dessins exécutés en couleurs naturelles; tandis que celui de Bologne n'en présente que 51 tous faits à la plume. Onze des derniers douze dessins du texte de Providence manquent dans celui de Bologne; mais il est évident que l'illustrateur de Bologne avait l'intention de les inclure, et même d'ajouter une dizaine d'autres qui manquent dans les deux manuscrits, mais sont annoncés dans le texte, puisqu'on trouve dans chaque cas un espace en blanc, parfois même encadré, qui était évidemment réservé pour le dessin: preuve que l'illustration du manuscrit ne fut pas achevée. En revanche, dans le manuscrit de Providence, nous ne trouvons pas d'espace vide là où le texte annonce un dessin qui ne fut pas exécuté, et aucun intervalle ne sépare le paragraphe précédent de celui qui suit. Il semblerait 
que dans ce manuscrit l'illustrateur ait précédé le copiste, tandis que nous avons l'impression contraire avec celui de Bologne. Dans l'un comme dans l'autre, le texte du Brief Discours est complet, mais le dessinateur n'a pas achevé sa tâche. Le fait que neuf cartes et le croquis du requin soient annoncés dans le Brief Discours mais ne figurent dans aucun des manuscrits nous porte à croire que le texte original contenait au moins 72 cartes et dessins.

\section{LES SOURCES}

Champlain a-t-il dit la vérité ? Si nous voulons résoudre cette question, il nous faut faire une étude approfondie des sources. Dans ce but, le Brief Discours peut se diviser en trois parties de longueur inégale.

A) La première a rapport au traité de Vervins, et au rapatriement des troupes espagnoles sur des bateaux français. La correspondance des ambassadeurs espagnols avec leur gouvernement nous donne à ce sujet des renseignements que l'on trouve aux Archives de Simancas, dans la Section Estado $K$. Cette section est une de celles que Napoléon fit transporter en France en 1810. Le gouvernement du Maréchal Pétain l'a rendue à l'Espagne en 1942.

B) La seconde a trait au voyage de Blavet à Cadix, qui se fit avec la flotte de Pedro de Zubiaur, une des escadres de l'Armada del Mar Oceano. Cette armada fut créée en 1595. La Section Guerra Antigua des archives de Simancas contient un certain nombre de documents sur l'escadre de Zubiaur. On pourrait sans doute en trouver beaucoup plus dans l'Archivo del Mar Oceano à Sanlucar de Barrameda ; mais les ducs de Medina Sidonia, propriétaires de cet archivo, n'en facilitent point l'accès aux historiens, bien au contraire.

C) La partie la plus importante du Brief Discours se rapporte à l'armada de Francisco Coloma qui fit voile vers l'Amérique en 1599 et revint l'année suivante. On pourrait facilement écrire une histoire de cette flotte en dix volumes, sans épuiser l'énorme documentation que nous offrent à ce sujet les Archives des Indes à Séville, particulièrement les Sections III (Contratación) et V (Indiferente General). 


\section{De Blavet À Cadix}

Champlain raconte que pendant la guerre de la Ligue, il avait servi comme maréchal des logis dans l'armée française qui opérait en Bretagne. Le traité de Vervins (2 mai 1598) le laissa sans emploi. "Je me résolus pour ne demeurer oisif, de trouver moyen de faire ung voiage en Espagne, en y estant pratiquer et acquérir des cognoissances pour... pouvoir m'embarquer dans quelqu'un des navires de la flotte que le Roy d'Espaigne envoye tous les ans aux Indes Occidentalles affin d'y pouvoir m'y informer des particularités qui n'ons peu estre recognues par aucun Françoys à cause qu'ilz n'y ont nul accez libre pour à mon retour en faire rapport au vray à sa Majesté. »

Ayant ainsi expliqué le but de son voyage, il ajoute: "Pour donc parvenir à mon desseing, je m'en allay à Blavet où lors il $\mathrm{y}$ avoit garnison d'Espaignolz, auquel lieu je trouvay ung mien oncle nommé le Cappitaine Provençal, teneu pour ung des bons mariniers de France, et qui en ceste qualité avoit esté entreteneu par le Roy d'Espaigne comme pillotte général en leurs armées de mer. Mondict oncle ayant receu commandement de monsieur le mareschal de Brissac de conduire les navires dans lesqueiz l'on feist embarquer les Espaignolz de la garnison dudict Blavet, pour les repasser en Espaigne, ainsy qu'il leur avoit esté promis, je m'embarquay avec luy dans un grand navire du port de cinq centz thonneaux nommé le Saint Julian qui avait esté pris et arresté pour ledict voiage. »

Quels renseignements les sources espagnoles peuvent-elles nous fournir sur le Saint Julian, et sur le Capitaine Provençal, oncle de Champlain?

Le traité de Vervins avait décidé l'évacuation du territoire français par les troupes du Roi Catholique. La forteresse de Blavet (Port-Louis) qui avait été quartier général espagnol pendant la guerre, devait être démantelée, sa garnison et son artillerie ramenées en Espagne. Dans ce but on avait envoyé en Bretagne Pedro de Zubiaur avec une petite flotte. Zubiaur arriva à Blavet le 3 juillet 1598. Très vite il se rendit compte que ses trois hourques étaient insuffisantes pour procéder à l'évacuation, et d'accord avec Cossé Brissac, gouverneur français de Bretagne, 
il chercha à se procurer d'autres vaisseaux. Cossé Brissac avait reçu à cet effet pleins pouvoirs du gouvernement espagnol, et le 15 juillet un contrat fut passé entre « Haut et Puissant Seigneur Messire Charles de Cossé, comte de Brissac, maréchal, grand pannetier, grand fauconnier de France, lieutenant général du Roy en ces pays de Bretaigne, d'une part, et l'écuyer Julien de Montigny, seigneur de la Haultière, gentilhomme ordinaire de la chambre du Roy, resident en cette ville de Vannes....» pour le fret de deux navires de La Hautière, le Saint Julian, de 500 tonnes, et le Jacques (ou Saint Jacques), embarcation plus petite de 100 tonnes. $^{5}$

La Hautière est un personnage intéressant. Il avait pris une part active aux guerres de la Ligue; lui et son beau-frère René d'Aradon, gouverneur de Vannes, avaient été les chefs du parti catholique en Bretagne méridionale. En 1595, au cours d'un voyage en Espagne, il avait eu une entrevue avec Philippe II. ${ }^{6}$ La Hautière s'intéressait aussi aux pêcheries de Terreneuve. A l'époque de la paix de Vervins, il armait deux navires qu'il projetait d'envoyer d'abord aux Canaries, puis à Terreneuve. Ce furent précisément ces deux vaisseaux que Brissac fréta au nom du Roi Catholique.

Suivant les termes du contrat, La Hautière devait recevoir 10 livres (40 réaux) par tonne, mais devait prendre à sa charge la manutention des équipages. Les bateaux devaient partir dans les trois jours, ne pas dépasser le Cap Finisterre, ne pas rester plus de six jours dans le port où ils débarqueraient les troupes et l'artillerie ramenées de Blavet, et prendre immédiatement le chemin du retour. Sébastian Zamet, banquier florentin et agent financier du roi d'Espagne à Paris, garantit l'exécution du contrat, qui subit quelques entorses comme nous le verrons bientôt.

Quant à l'oncle de Champlain, le Capitaine Provençal, il n'était pas pilote général de l'armada espagnole, comme l'affirme l'auteur du Brief Discours, mais bien capitaine du Saint-Julian. J'ai trouvé son nom dans quatre documents de l'Archivo de Indias et dans six autres à Simancas, sous les formes suivantes:

5 Simancas, Estado K. 1602, B, 86, Num. 32.

6 Simancas, Estado K. 1596, B, 83, Num. 50 a. 
Guillermo Eleno, Guillermo Eleno Provençal, Capitán Provençal et Capitán Provenzano. ${ }^{7}$ Son nom authentique était Guillaume Hellaine, « Provençal » n'étant qu'un surnom. Tout comme Champlain, semble-t-il, il était naturel de Brouage. En 1600, il disait avoir soixante ans. Nous aurons lieu d'étudier plus loin ses rapports avec Champlain.

En somme, le Brief Discours s'accorde assez bien avec les sources espagnoles. Le Saint Julian fit voile en août, date indiquée par Champlain. Suivant les termes du contrat passé avec La Hautière le 15 juillet, l'escadre devait partir dans les trois jours. Mais plusieurs semaines furent nécessaires pour démanteler le fort et trouver un nombre suffisant de navires pour le transport des troupes et de l'artillerie. Enfin Pedro de Zubiaur put mettre à la voile le 23 août 1598 avec 18 vaisseaux, presque tous français.

La flotte arriva à Vigo le 28, où Zubiaur reçut quelques jours plus tard l'ordre de continuer jusqu'à Cadix. Parti de Vigo le 7 septembre, il entra une semaine plus tard dans Cadix (14 Septembre)..$^{8}$

L'auteur du Brief Discours n'a pas grand chose à dire sur la traversée de Blavet à Cadix. Il mentionne une tempête qui dispersa la flotte à la hauteur du Cap Finisterre. Cette tourmente fut peut-être la cause de la séparation du Jacques du reste de la flotte, car ce navire ne dépassa pas Lisbonne où il débarqua son chargement d'hommes et d'artillerie. Par contre le Saint Julian entra dans Cadix avec le reste de l'escadre.

Le Brief Discours ne mentionne pas une épidémie qui décima les équipages. Peut-être le Saint Julian fut-il épargné. Zubiaur écrit: "J'ai jeté à la mer de mes propres mains le cadavre d'un gentilhomme irlandais qui mourut sur ma capitane. ${ }^{9}$ Un des navires français, la Maria, du Capitaine Combas, servit d'hôpital pendant la traversée depuis Vigo. Zubiaur le fit brûler et couler

7 A.G.I. (Archivo General de Indias), Contaduria 336, Contratación 2957, 3656, 3968.

Simancas, Estado K 1451, A 58. num. 1; Estado K 1603, N. 86, num 107; Guerra Antigua 567 et 572; Contaduria Mayor (2da. Epoca), 406.

8 A.G.I., Contratación 5112. (Zubiaur, 12 et 15 sept. 1598).

9 Simancas, Guerra Antigua 529. (7 oct. 1598). 
dans la baie de Cadix pour éviter toute contagion ultérieure. Combas fut indemnisé trois ans plus tard pour la perte de son vaisseau. ${ }^{10}$

Champlain écrit: «Seiournames audict lieu de Calis un moys entier, durant lequel j'eu le moyen de reconnoitre l'isle dudict Callis ... \ Les sources espagnoles nous confirment que le Saint Julian resta ancré dans la baie de Cadix du 14 septembre au 12 octobre.

\section{SANLUCAR DE BARRAMEDA}

La veille de l'arrivée de Zubiaur à Cadix, on apprit en Espagne la prise de Porto Rico par le comte de Cumberland, ce qui constituait un sérieux revers, car la perte de l'île représentait un danger permanent pour les cargaisons de métaux précieux que les armadas du Roi Catholique ramenaient tous les ans du Pérou et de la Nouvelle-Espagne. Il fallait à tout prix reconquérir Porto Rico; mais pour cela une flotte considérable était nécessaire. On attendait précisément à cette époque le retour de l'escadre des Açores, commandée par Joanes de Urdayre qui escortait la flotte revenant de la Nouvelle-Espagne. Il fut décidé que les dix galions de Urdayre, renforcés par les bateaux français de Zubiaur, formeraient la nouvelle armada, et que les troupes et l'artillerie ramenés de Blavet serviraient à la reconquête de Porto-Rico. Fin septembre, Zubiaur fut nommé capitaine général de l'armada et Urdayre lui fut adjoint comme amiral. ${ }^{11}$

Cette armada ne devait constituer que la moitié d'une énorme expédition militaire, car à Lisbonne on équipait une autre flotte sous la direction de Diego Brochero de Anaya. Les deux flottes devaient traverser séparément l'Atlantique et se joindre dans les parages de la Guadeloupe, où Brochero prendrait le commandement suprême des opérations contre Porto-Rico. ${ }^{12}$

Zubiaur commença immédiatement à choisir entre ses navires ceux qui étaient les plus propres à la nouvelle entreprise. Un de ceux qu'il retint dans ce but était le Saint Julian. C'était évi-

10 Simancas, Guerra Antigua 585.

11 A.G.I., Indiferente General 1866.

12 Simancas, Guerra Antigua 563 ( 7 nov. 1598). 
demment une violation du contrat passé avec La Hautière à Vannes. Quand ce dernier intenta plus tard un procès au gouvernement espagnol, Zubiaur s'excusa en affirmant que le Saint Julian n'appartenait pas réellement à La Hautière, mais à un $M$. de Landricart à qui La Hautière l'avait enlevé pendant les guerres de la Ligue. Zubiaur ajouta que le capitaine du Saint Julian, Guillermo Eleno, avait reçu pleins pouvoirs de Landricart, et que c'était en vertu de ces pleins pouvoirs que le Saint Julian avait été frété pour servir en Amérique. ${ }^{13}$

Je doute fort que Zubiaur ait cru lui-même à cette histoire qu'il paraît avoir inventée pour les besoins de la cause. Dans une lettre adressée au Conseil des Indes, le 12 octobre 1598, parmi les navires réquisitionnés par lui il nomme «le Saint Julian de la Otier de Francia ». ${ }^{14}$ A cette époque il semble done qu'il n'avait aucun doute sur la propriété du navire. Le même jour, les bateaux choisis par Zubiaur furent envoyés à Sanlucar pour carénage.

Voyons maintenant ce que dit Champlain de son séjour à Sanlucar. "Partant dudict Calix nous fusmes à St. Luc de Barrameda qui est à l'entrée de la rivière de Siville, où nous demeurames troys moys, durant lesquelz je feuz à Siville, en prix le plan de l'une et de l'autre, que j'ay jugé à propos de representer au mieux qu'il m'a esté possible en ceste page et en la suivante. » Ces deux cartes sont exactes et semblent l'œuvre de quelqu'un qui a été sur place. Cependant sur la carte du manuscrit de Providence qui représente l'embouchure du Guadalquivir, on relève l'erreur suivante: le fameux monastère de Nuestra Señora de la Regla est placé au sud de Rota, alors qu'il se trouve au nord, à moins que l'auteur de la carte n'ait confondu Rota avec Chipiona.

Champlain ne paraît avoir eu qu'une idée assez confuse de l'expédition projetée. Il semble croire que l'armada de Zubiaur fut organisée pour défendre Porto-Rico contre les Anglais et que le projet fut abandonné lorsque ces derniers s'emparèrent de l'île: «Il arriva des nouvelles par une patache d'advis qu'il avois esté pris des Anglais; au moien de quoy le dict voiage fut rompu à mon grand regret pour me voir frustré de mon espérance. » Ce

13 Simancas, Estado K 1603, B 86. num. 107.

14 A.G.I., Indiferente General 1114. 
fut bien au contraire la perte de l'île et le désir de la reconquérir qui motivèrent les plans de l'expédition Brochero-Zubiaur. Si le projet fut abandonné plus tard, c'est que les Anglais avaient déjà évacué Porto-Rico. Le danger était passé, et il n'était plus besoin d'une entreprise de telle envergure.

La rumeur que le Comte de Cumberland avait évacué l'île circulait à Séville dès la fin d'octobre 1598 , mais on n'en obtint confirmation officielle que le 20 novembre: la flotte anglaise décimée par une épidémie avait abandonné Porto-Rico le 27 août. ${ }^{15}$ On annula aussitôt le départ de l'escadre de Diego Brochero qui s'équipait à Lisbonne; quant à la flotte de Zubiaur qui se trouvait à Sanlucar, elle changea complètement de caractère. On la transforma en l'armada que l'on envoyait annuellement en Amérique pour en ramener les métaux précieux. Pour cette raison probablement, Zubiaur fut relevé de son commandement et on lui substitua Francisco Coloma (25 décembre). Urdayre resta comme amiral. ${ }^{16}$

Zubiaur s'était rendu célèbre comme corsaire en luttant contre les Anglais, les Hollandais et les Huguenots français, mais il n'avait aucune expérience de l'Amérique. Coloma au contraire avait déjà servi aux Indes et avait été nommé général titulaire de l'Armada de la Route des Indes en 1592. En 1594-95, il avait eu sous ses ordres trois flottes combinées: la sienne propre, et celles de Nouvelle-Espagne et de Terre Ferme; mais, enclin à remplir ses poches, il avait commis tant d'abus qu'on l'avait suspendu pour trois ans. Cette période passée, il rentrait en grâce et on lui redonnait un commandement. En parfait courtisan, il écrivait au jeune Philippe III, monté sur le trône trois mois plus tôt: «Je ne doute pas du grand succès de cette armada, car je suis le premier capitaine général nommé par Votre Majesté. »17

Zubiaur ne fut officiellement informé de la perte de son commandement que le 13 janvier 1599. Il dut en souffrir, car il s'était montré fort désireux d'aller en Amérique. Il avait même fait

15 A.G.I., Contratación 5170 (f. 320).

16 A.G.I., Indiferente General 2496 (f. 219-231), 3264 A.

17 Simancas, Guerra Antigua 556. Francisco Coloma était le fils du comte d'Elda, et le frère du diplomate et historien Carlos Coloma. Il mourut en décembre 1600 . 
charger sur le Saint Julian une cargaison de vin et d'huile sans en demander l'autorisation à la Maison de la Contratación. Ce chargement de contrebande devait causer bien des ennuis aux officiers du Saint Julian. Plusieurs furent condamnés plus tard pour vente illégale de vin et d'huile en Amérique. ${ }^{18}$

Après Zubiaur, vint le tour de Guillermo Eleno. Quelques jours avant le départ de l'armada, Francisco Coloma le remplaça comme capitaine du Saint Julian par Yeronimo de Vallebrera, un officier qui avait servi sous lui en 1594-95. Champlain veut nous faire croire que son oncle fut retenu par Zubiaur pour servir ailleurs, et ne put à cause de cela partir pour l'Amérique. Mais la vérité est toute autre. Zubiaur et Eleno furent destitués tous les deux sans recevoir aucun autre commandement. «Je m'occuperai de vous selon vos mérites quand l'occasion s'en présentera » écrivait le roi à Zubiaur en lui annonçant sa destitution. ${ }^{19}$ Zubiaur et Eleno restèrent à Sanlucar, aidant à équiper la flotte de Nouvelle-Espagne qui mit à la voile en juin 1599 ; puis Zubiaur fut nommé général de l'escadre du Détroit de Gibraltar et choisit Eleno pour commander un de ses navires. La preuve de la destitution d'Eleno par Coloma se trouve dans deux documents des Archives des Indes, datés tous les deux du 26 février 1599. Ce jour-là, à Seville, Eleno recevait 300 réaux de la Maison de la Contratación «comme accompte sur la solde qui lui est due pour avoir servi comme capitaine du Saint Julian, un des vaisseaux que le général Pedro de Zubiaur a ramenés de Bretagne, depuis le 11 octobre de l'année passée qu'il entra à Sanlucar et commença à servir dans l'armada qui devait aller à Porto-Rico, jusqu'à fin de janvier de cette année, quand le général Don Francisco Coloma, qui mena ledit navire aux Indes avec son armada, y nomma un capitaine $\gg^{20}$

Dans quelle mesure ces changements affectèrent-ils Champlain ? «Le Capitaine Provençal, mon oncle, ayant esté reteneu par le général Soubriago pour servir ailleurs, et ne pouvant faire le voyage, me commit la charge dudict vaisseau pour avoir esgard

18 A.G.I., Escribania de Cámara 1012 A.

19 A.G.I., Indiferente General 2496 (f. 39 ).

20 A.G.I., Contratación 2957, Contaduria 336. 
à iceluy, que j'acceptay fort vollontiers, et sur ce nous fusmes trouver ledict général Colombe pour savoir s'il auroit agréable que je fisse le voiage; ce qu'il me promist librement, avec des temoignages d'en estre fort aise, m'ayant promis sa faveur en assistance, qu'il ne m'a depuis desniés aux occasions. »

L'auteur du Brief Discours cherche évidemment à se donner une importance qu'il n'avait pas. Pour qu'on lui eût commis la charge du Saint Julian, il lui aurait fallu être un des trois officiers supérieurs du navire: capitaine, maître ou pilote; or il n'était ni l'un ni l'autre. Morris Bishop croit qu'il dut faire le voyage comme subrécargue et représentant des armateurs. Mais Francisco Coloma affirme que tel représentant n'existait pas. Dans un rapport adressé au Conseil d'Etat le 5 Octobre 1600 à propos de la vente du Saint Julian à La Havane, le général écrit: «... et comme il n'y avait personne qui eût procuration du propriétaire, j'ordonnai aux officiers de faire un inventaire détaillé $\gg .{ }^{21}$ Donc, aucun doute, Champlain n'eut pas le vaisseau à sa charge.

A-t-il fait le voyage comme officier subalterne ? Nous connaissons les noms de six des officiers du Saint Julian (capitaine, maître, pilote, contremaître, quartier-maître, écrivain). Tous ont des noms espagnols, excepté le contremaître qui était probablement italien. Manquent les noms des officiers subalternes suivants: charpentier, tonnelier, calfat, dépensier, barbier-chirurgien, alguacil del agua..2 Champlain fut-il l'un d'entre eux ?

Il est plus probable qu'il a fait le voyage comme simple marin ou comme passager clandestin. De nombreux matelots étrangers servaient dans l'armada bien qu'ils n'y eussent été admis qu'à contre-cœur, car on craignait que les connaissances acquises par eux aux Indes ne les induisent à y revenir plus tard comme corsaires ou comme contrebandiers. "Ils se font tant experts qu'ils connaissent tous les recoins mieux que ceux de leur pays » écrivait Coloma qui ne tenait nullement à les engager ; ${ }^{23}$ mais comme

\footnotetext{
21 Simancas, Estado K 1603, B 86, num 115. d'eau.

22 L'alguacil del agua était l'officier chargé de distribuer les rations

23 A.G.I., Indiferente General 1115. (3 janvier 1599).
} 
on manquait de marins espagnols, il fallut bien en passer par là. L'admission des étrangers fut longtemps discutée par les dirigeants de la Maison de la Contratación. Finalement on reçut des ordres de Madrid permettant l'engagement des étrangers à l'exception des Anglais et des Hollandais rebelles. Le Saint Julian dut garder un grand nombre de marins français, mais comme le navire fut vendu à La Havane, le rôle de son équipage n'est pas conservé. Nous connaissons cependant les noms de plusieurs matelots du Saint Julian qui, après la vente du navire, commencèrent à servir sur d'autres vaisseaux et revinrent ainsi en Espagne. ${ }^{24}$

Peut-être Champlain entreprit-il le voyage comme passager clandestin, ainsi que devait le faire Thomas Gage 25 ans plus tard. Un grand nombre de "passagers tombés du ciel » voyageaient à bord des flottes espagnoles, car l'autorisation de passer aux Indes était fort difficile à obtenir pour les Espagnols, et presque impossible pour les étrangers. Mais avec de l'argent on résout bien des problèmes, et les passagers clandestins constituaient une source de revenus fort appréciable pour les capitaines. Nous savons qu'au moins six d'entre eux voyageaient sur le Saint Julian introduits tous à bord par Yeronimo de Vallebrera. Les quatres premiers étaient des gentilhommes espagnols qui mangeaient à la table du capitaine. Le cinquième était un tailleur nègre. Le dernier, natif de Triana et nommé Francisco de Sequera, figurait comme marin sur le registre de l'équipage, mais c'était Vallebrera qui empochait sa solde. Sequera n'était d'ailleurs nullement marin et remplissait à bord les fonctions de domestique. Accusé plus tard d'homosexualité, il fut mis aux fers puis jugé à Vera-Cruz. Ce fut lui qui, au cours de son procès, révéla la présence des autres « passagers tombés du ciel » à bord du Saint Julian. ${ }^{25}$

Selon le Brief Discours, l'armada quitta Sanlucar de Barrameda au début de janvier 1599; mais toutes les sources espagnoles donnent comme date de départ le 3 février. Ce jour-là, un pilote de Sanlucar, nommé Adrián García, fit franchir au Saint Julian la barre qui se trouve à l'embouchure du Guadalquivir;

24 A.G.I., Contratación 2958.

25 A.G.I., Contratación 64. 
après quoi le navire mit à la voile avec le reste de la flotte. García toucha dix ducats pour sa peine. ${ }^{26}$

Avant le départ, Coloma reçut de Madrid des instructions fort détaillées. De Sanlucar, il devait aller directement à PortoRico et y débarquer le nouveau gouverneur et les 400 soldats qui devaient former la garnison. Puis il devait diviser son armada en deux escadres: l'une d'elles, sous son propre commandement, devait faire voile pour Carthagène et Porto-Belo; l'autre, à la charge de Joanes de Urdayre, suivrait la côte nord de St-Domingue pour la nettoyer des navires ennemis qui l'infestaient, et prendrait ensuite le chemin de Vera-Cruz.

\section{De SANLUCAR À Vera-CruZ}

Le récit que fait Champlain du voyage de Blavet à Cadix paraît avoir été vécu; mais après que l'armada quitte Sanlucar, le Brief Discours devient si vague qu'on n'y trouve pas une seule fois le nom d'une seule personne ou d'un seul navire, pas même du Saint Julian. L'on n'y rencontre non plus aucune date précise, mais seulement des remarques fort vagues, telles que «nous restâmes ici un mois » ou « nous arrivâmes après six jours ».

Suivons l'itinéraire de l'armada. Après avoir laissé les Canaries au sud, on traversa l'Atlantique et on arriva en vue de la Désirade «qui est la premiere ille qui faut que les pilottes recognoissent nécessairement pour aler en toutes les autres illes et portz des Indes ». Cette remarque est très vraie, mais l'auteur du Brief Discours se trompe quand il affirme que la traversée s'effectua en deux mois et six jours. L'armada couvrit la distance de Sanlucar à la Désirade en un mois et deux semaines, du 3 février au 18 mars.

Champlain ajoute ensuite: "De la dicte isle nous feusmes a une autre isle nommée la Guadalouppe qui est fort montaigneuse, habitée de sauvages, en laquelle il y a quantité de bons portz, a l'un desquelz, nommé Nacou, nous fusmes prendre de l'eau, et comme nous mettions pied à terre, nous veismes plus de trois centz sauvaiges qui s'en fuirent, dedans les montaignes sans qu'il fust à notre puissances d'en attraper un seul, estant plus dispotz à la

26 A.G.I. Contratación 3656 . 
course que toux ceux des nostres qui les voulurent suivre. Ce que voiant, nous en retournasmes dans nos vaisseaux après avoir pris de l'eau et quelques refreschissementz, comme chair et fruictz de plaisans goust. Ceste isle contient environ vingt lieux de long et douze de large, dont la forme est telle que la figure suivante. »

Cette description est fort attrayante, mais à quel point estelle vraie? Sans doute les armadas qui se dirigeaient sur VeraCruz ou Carthagène avaient l'habitude de mouiller à la Guadeloupe pour y renouveler leur provision d'eau; mais Coloma ne le fit pas parce qu'il devait faire escale à Porto-Rico, à quatre jours seulement de la Guadeloupe, et parce qu'il avait ordre de se hâter. Voici ce qu'il écrivit de Porto-Rico le 27 mars: «J'ai suivi fidèlement les ordres de V. M. de ne perdre une seule heure de temps, et quoique les galions d'armada avec leur cargo humain soient généralement obligés de faire l'aiguade à la Guadeloupe, je m'en suis dispensé puisque je devais arriver ici ».27 Coloma confia sa lettre à un capitaine de patache, Juan de Ojeda. Lorsque ce dernier arriva à Séville et qu'on l'interrogea sur le sort de l'armada, il répondit: « Tout s'est bien passé, quoiqu'il ait fallu attendre l'hourque Ciervo Volante; en somme tout s'est bien passé, et ils sont arrivés à Porto-Rico en 49 jours, sans toucher à aucune terre. ${ }^{28}$ Donc aucun doute, L'armada de Coloma n'a pas ancré à la Guadeloupe, et Champlain n'a pas pu y débarquer.

La carte de l'île que l'on trouve dans le Brief Discours montre beaucoup d'imagination. Son auteur ne sait pas apparemment que la Guadeloupe est divisée en deux îles séparées par un étroit canal. Le dessin de la baie où ancraient les navires, que Champlain appelle Nacou, est également fantaisiste. Nous avons plusieurs descriptions de ce mouillage, où se jetaient quatre petits ruisseaux dont l'eau servait à faire l'aiguade. L'endroit avait été baptisé solennellement en août 1594 «Puerto de Santa Ana de la Ysla de Guadalupe ${ }^{29}$

27 A.G.I., Indiferente General 745.

28 A.G.I., Indiferente General 1115 (6 mai 1599).

29 Voir les descriptions de Luis Alfonso Flores (30 août 1594). A.G.I., Contratación 5110; Juan de Salazar (1603), publiée dans la Colección de Documentos inéditos para la Historia de España, LII, 459 ss; et le manuscrit de Nicolás de Cardona qui se trouve à la Bibliothèque Nationale de Madrid, et qui contient un croquis du mouillage. 
Reprenons maintenant le voyage à Porto-Rico, tout en suivant l'itinéraire du Brief Discours. "Aprés avoir demeuré deux jours au dict port de Nacou, le troisièsme jour nous nous remismes a la mer, et passasmes entre des isles que l'on nomme las virgines ... D'icelles isles nous feusmes à l'isle de la Marguerite où se peschent les perles. » L'énorme bourde, qui consiste à placer la Marguerite entre les Iles Vierges et Porto-Rico a déjà été relevée par Morris Bishop. Qui a pu induire Champlain à commettre une telle erreur ? Peut-être le fait que quand une armada arrivait en vue de la Désirade, son général dépêchait toujours un bateau à la Marguerite - qui se trouve en réalité près de la côte du Vénézuéla - pour y recueillir les perles qui s'y pêchaient. Le vaisseau que Coloma y avait envoyé était une patache nommée Sandoval.

Enfin nous arrivons à Porto-Rico. Champlain en raconte la capture par les Anglais d'une façon assez exacte. La conduite du gouverneur espagnol fut jugée sévèrement par ses propres compatriotes, bien que le Conseil des Indes l'ait blanchi plus tard. Par contre, l'auteur du Brief Discours suit de fort loin la vérité lorsqu'il traite de l'état de l'île à l'arrivée de l'armada. Selon lui, les Anglais ne l'avaient évacuée que quinze jours avant, les habitants vivaient cachés dans la montagne, et la ville était déserte à l'exception de quelques nègres et indiens. Nous avons déjà remarqué que la rumeur de l'évacuation de l'île circulait en Espagne dès la fin d'octobre 1598. Les Anglais avaient abandonné Porto-Rico le 27 août, c'est-à-dire sept mois avant l'arrivée de l'armada, et non quinze jours comme le prétend Champlain. Lorsqu'il débarqua, Coloma trouva dans la ville 280 habitants mâles ainsi qu'un gouverneur intérimaire envoyé de St-Domingue, qui s'appelait Yeronimo de Agüero Vardezi. Ce dernier avait déjà commencé la reconstruction $d u$ fort et $\mathrm{y}$ avait fait monter treize canons abandonnés par les Anglais. ${ }^{30}$

Champlain écrit: "Nous demeurames audict Portorico environ un moys. » Mais toutes les sources espagnoles affirment que l'armada n'y resta que dix jours, du 22 au 28 mars.

30 Ojeda, 6 mai 1599. A.G.I., Indiferente General 1115. Le nom de Agüero Vardezi se trouve dans une lettre d'Alonso de Mercado, A.G.I., Escribania de Cámara 971 . 
Pendant son court séjour à Porto-Rico, Coloma désembarqua les 400 soldats qui devaient former la garnison (300 selon Champlain). Il fit aussi prêter le serment d'usage au nouveau gouverneur Alonso de Mercado. ${ }^{31}$ Le 27 mars le général divisa son armada en deux escadres. La sienne devait faire voile pour Carthagène, puis pour Porto-Belo, afin d'y charger l'or et l'argent qui venaient du Pérou par l'isthme de Panama; tandis que l'autre escadre, sous le commandement de Joanes de Urdayre mettrait le cap sur Vera-Cruz. Cette dernière escadre comprenait deux des six galions, le Saint Julian qui avec ses 500 tonnes était du port d'un galion, et trois vaisseaux plus petits. Les deux flottes partirent ensemble le lendemain et se séparèrent en haute mer quelques heures plus tard.

Avant de quitter Porto-Rico, Coloma transmit à Urdayre l'ordre de nettoyer la côte nord de St-Domingue des navires ennemis qui l'infestaient. Cela n'obligeait nullement l'amiral à changer de direction, puisque la route de Porto-Rico à Vera-Cruz côtoie le nord de St-Domingue. A cette époque, toute la partie de la côte comprise entre Puerto Plata et La Yaguana était fréquentée par des corsaires anglais, hollandais et français qui fournissaient aux Espagnols du drap et des nègres, en échange de cuirs et de mélasse, car il fallait des esclaves pour le travail des plantations. Tout commerce avec l'étranger était interdit sous la menace des peines les plus sévères, ce qui n'empêchait pas les habitants d'accueillir les marchands protestants à bras ouverts. Les contrebandiers pouvaient en plus compter sur la complicité de quelque deux cents Espagnols en rupture de ban qui avaient trouvé refuge dans les forêts. En fait les étrangers se sentaient si bien chez eux qu'ils avaient leurs forges, leurs chantiers, leurs foyers dans St-Domingue. Coloma rapporte avec dégoût que «la femme d'un des principaux habitants ayant accouché, ce fut un corsaire luthérien français qui tint l'enfant sur les fonts baptismaux et donna une grande fête à cette occasion ${ }^{32}$

31 Voir l'acte dressé par l'écrivain chef de l'armada, Bartolomé Martinez de Forné. A.G.I. Indiferente General 1116. (15 février 1600).

32 Coloma, juin 1600. A.G.I., Indiferente General 1289. 
L'auteur du Brief Discours suit avec exactitude l'itinéraire de Joanes de Urdayre, et ses croquis de Puerto Plata, Manzanillo, Mosquitos et Monte Christi semblent pris sur le vif ou sont d'excellentes copies. Il mentionne dans son récit la montagne blanche et brillante qui se trouve derrière Monte Christi et qui sert encore de point de repère aux navigateurs; mais il se trompe en plaçant Monte Christi entre Mosquitos et Saint Nicolas, alors que ce port est beaucoup plus à l'est entre Puerto Plata et Manzanillo. Pour se rendre de Mosquitos à Monte Christi, il aurait fallu faire demitour, ce qui aurait entraîné la perte d'une semaine au moins, à cause des vents contraires de l'est qui dominent dans cette région, bien que l'auteur du Brief Discours veuille nous faire croire que la distance aurait été couverte en un seul jour. Il est parfaitement clair que la flotte n'est pas revenue sur ses pas et que Champlain a brouillé l'ordre géographique des ports de la Côte nord de St-Domingue.

Nous avons la preuve documentaire du passage de l'escadre au large de Manzanillo. ${ }^{33}$ La poursuite de deux bateaux français près de Mosquitos nous est aussi confirmée par d'autres sources. L'un des vaisseaux se brisa contre les récifs, et l'autre qui venait de Dieppe fut capturé. Champlain et les sources espagnoles sont d'accord à ce sujet. Le bateau pris par Urdayre fut mené à Vera-Cruz où il fut rebaptisé San Juan de la Presa. ${ }^{34}$

Nous arrivons maintenant à l'épisode le plus dramatique du Brief Discours: la rencontre avec la flotte franco-anglo-hollandaise sur la côte occidentale de St-Domingue. La version qu'en donne Champlain s'accorde avec les sources espagnoles, à l'exception de quelques menus détails, tels que le nombre des navires ennemis (treize selon Champlain, au lieu de onze), et l'emplacement de l'escarmouche. Le Brief Discours la situe près du Cap Saint-Nicolas, tandis que les sources espagnoles la placent à Gonaives. Voyons maintenant ce qui eut lieu.

Le soir du 7 avril, l'escadre de Joanes de Urdayre arriva au large de Gonaives. Dans la baie se trouvaient ancrés onze navires

${ }^{33}$ A.G.I., Audiencia de Santo Domingo 81. (papiers du capitaine Alvaro Paredes Carreño).

34 Urdayre, 4 mai 1599. A.G.I., Contratación 2965. 
avec une poignée d'hommes à bord, car la plus grande partie des équipages était à l'intérieur, occupée à commercer avec les Espagnols. Quelle proie facile pour l'escadre de Urdayre que ces vaisseaux presque sans défense ! Malheureusement le vent était contraire et ne permettait pas d'entrer dans la baie, ce qui obligea l'amiral à attendre au lendemain. Ce délai fut mis à profit par les chefs protestants, qui s'étaient aussi rendu compte que le seul navire espagnol dessus le vent, le galion San Giuseppe s'était échoué sur un bas-fond. Sans attendre leurs compagnons restés à l'intérieur de l'île, ils mirent à la voile au petit jour et passèrent sous le nez de la flotte espagnole malgré une vive canonnade. Le San Giuseppe finit par se remettre à flot, et l'amiral ordonna la poursuite; mais il était trop tard. Au bout de quelques heures, Urdayre se rendit compte qu'il n'avait aucun espoir de rejoindre les fuyards beaucoup plus rapides que lui, et il abandonna l'entreprise.

Le Brief Discours ne mentionne pas l'accident survenu au San Giuseppe. Champlain se contente d'accuser l'amiral et ses hommes de manque de courage. Mais dans leurs rapports, Coloma et Urdayre ne manquèrent pas de blâmer les deux capitaines du galion, le capitaine de mer Miguel Cerdán et le capitaine d'infanterie Francisco de Venegas. Après le retour de l'armada, ces deux derniers furent déclarés coupables et privés de leur commandement. ${ }^{35}$

En plus des croquis de Puerto Plata, Manzanillo, Mosquitos et Monte Christi, le Brief Discours contient deux autres cartes qui ont trait à St-Domingue. Dans l'une apparaît l'île entière. L'autre, intitulée «le port de la Yaguana » représente en réalité toute la côte sud-ouest de St-Domingue, mais défigurée de telle façon qu'on dirait une petite baie. Le cap Tiburon y est appelé «Cap Saint-Nicolas ». L'île Caymitos, nommée correctement dans le manuscrit de Bologne, est appelée «Cayima » dans celui de Providence.

35 Coloma, 23 février 1600, A.G.I., Indiferente General 1116. Residencia de Francisco de Venegas, Miguel Cerdán y Joanes de Urdayre, 10 avril 1600, A.G.I., Escribanía de Cámara 972 et 967. 
Continuant le voyage, Champlain donne les Caymans comme l'escale suivante. Voici en forme condensée ce qu'il en écrit: "Laissant la dicte ille St. Domingue, nous continuasmes nostre route de la Neufve Espaigne... Nous allasmes cotoyer l'isle de Cuba à la bande du sud, terre assez haute. Nous allasmes reconnoistre de petits isles qui s'appelent les Caymanes, au nombre de syx ou sept... Nous mouillasmes l'ancre entre les isles et y fusmes ung jour. Je mis pied à terre en deux d'icelles et vis ung très beau havre fort agréable ....»

Les rapports de Joannes de Urdayre ne font mention d'aucune escale de son escadre aux Caymans. Il n'avait d'ailleurs aucune raison de la faire puisqu'il avait ordre de se hâter, et puisque les provisions d'eau et de combustible obtenus à PortoRico devaient largement lui suffire pour arriver à Vera-Cruz. Les Caymans manquaient d'ailleurs d'eau et de bois. La description qu'en fait Champlain, il a dû sans doute l'obtenir de quelque corsaire français qui les avait visités, car les Caymans servaient souvent de repaire aux aventuriers de haute mer. La carte qu'on en trouve dans le Brief Discours est assez fantaisiste. Elle représente sept îles très rapprochées, et les trois plus grandes portent des noms que je n'ai pu retrouver nulle part ailleurs. Les Caymans sont en réalité au nombre de trois, et la distance entre Grand Cayman et les deux autres dépasse 70 milles.

"Nous levames l'ancre le même jour au soir avec un fort bon vent et le lendemain sur les trois heures aprez midy nous arrivasmes a ung lieu qui s'appelle la Sonde, lieu très dangereux, car à plus de cinq lieues de là, ce ne sont que basses ... » Il fallut en effet un fort bon vent pour pouvoir parcourir en moins de 24 heures une distance de neuf degrés qui exigeait normalement une semaine de navigation.

Mais nous revenons à la réalité avec la description que Champlain fait de la Sonde, un étroit passage flanqué de dangereux récifs que les Espagnols appellent «Alacrans》, situés à une semaine de distance de Vera-Cruz. Dans le dessin à la plume du manuscrit de Bologne, la Sonde est fort nettement dessinée. La route normalement suivie par les armadas à cette époque de l'année passait au nord des «Alacrans 》, mais Urdayre se vit 
obligé de suivre le canal parce qu'il était pressé. Cependant avant d'y pénétrer, et pour dégager sa responsabilité, il demanda l'avis de tous ses pilotes, y compris celui de Francisco de Urbina, pilote du Saint Julian, et en fit prendre acte par l'écrivain chef de l'escadre. Tous les pilotes ayant donné avis conforme, la flotte s'engagea dans la Sonde le 24 avril..$^{36}$

« Huit jours après nous arrivasmes à St. Jean de Luz. » Tout à fait vrai, puisque l'escadre mouilla dans la baie de Vera-Cruz le $1^{\text {er }}$ mai 1599 . Champlain a fait un croquis remarquable de l'île de San Juan de Ulloa. Il y montre le quai construit par l'ingénieur Juan Bautista Antonelli où on amarrait les navires.

\section{VERA-CRUZ}

Nous arrivons maintenant à la partie du «Brief Discours 》 qui prête le plus à la controverse, puisque l'auteur prétend être allée à la cité de Mexico et plus tard à Porto-Belo, tandis que l'escadre restait ancrée dans la baie de Vera-Cruz. Considérons d'abord le temps qu'il lui aurait fallu pour faire ces deux voyages.

Voici ce qu'il en dit lui-même: "Quinze jours aprez nostre arrivée audict St. Jean de Luz, je m'en allay avec congé de nostre dict admiral à Mechique, distant dudict lieu de cent lieux... Aprez avoir demeuré ung mois entier à Mechique, je retournay a St. Jean de Luz, auquel lieu je m'enbarquay dans une patache qui alloit à Portovello... Nous feusmes trois semaines sur la mer avant d'arriver audict lieu de Portovello... Ayant demeuré ung moys audict Portovello, je m'en revins à St. Jean de Luz, où nous seiournasmes quinze jours, en attendant que l'on fist donner carenne à nos vaisseaux pour aller à La Havanne, au rendez-vous des armées et flottes. »

$\mathrm{Si}$ nous additionnons toutes ces données et ajoutons trois semaines de plus pour le retour de Porto-Belo à Vera-Cruz, nous obtenons un total de quatre mois et demi, durant lesquels l'escadre de Urdayre aurait dû rester ancrée à San Juan de Ulloa. Cette somme représente un strict minimum, puisqu'elle ne comprend pas le voyage d'aller et retour entre Vera-Cruz et Mexico; dis-

36 A.G.I., Contratación 64. 
tance que Champlain évalue à cent lieues, et puisqu'il faut aussi supposer qu'il soit parti pour Porto-Belo aussitôt après son retour de Mexico. Et pourtant ce strict minimum de quatre mois et demi est loin de concorder avec les 60 jours que l'escadre resta à Vera-Cruz, puisqu'étant arrivée le $1^{\text {er }}$ mai 1599 , elle mit à la voile le 29 juin de la même année. Il est évident que Champlain n'eut pas le temps matériel de passer un mois à Mexico et un autre à Porto-Belo. Il faut donc rejeter un de ces voyages, ou peut-être les deux. Etudions d'abord le voyage de Mexico.

Champlain affirme qu'il se rendit à la capitale de la Nouvelle Espagne « avec le congé de nostre dict admiral ». Par les comptes de Pedro de Mesa, payeur de l'escadre à Vera-Cruz, nous connaissons les noms des deux officiers que Urdayre envoya à Mexico. L'un d'eux était Yeronimo de Vallebrera, capitaine du Saint Julian; l'autre s'appelait Gaspar de Ubilla. Leur mission consistait à entrer en rapport avec le vice-roi pour accélérer l'envoi des métaux précieux à Vera-Cruz. Ils devaient aussi rapporter de Puebla de Los Angeles du biscuit et de la farine pour les équipages. Ils accomplirent leur mission en 39 jours. Sans doute ils ne firent pas le voyage seuls, car ils furent escortés par des guides que leur fournirent les autorités de Vera-Cruz. Mais personne d'autre ne fut envoyé à Mexico par Urdayre, à l'exception d'un courrier nommé Francisco Lopez Yarza. Donc, si l'auteur du Brief Discours a fait le voyage de Mexico, il a dû le faire sans la permission de l'amiral, ce qui n'aurait d'ailleurs rien d'impossible. Les statistiques montrent qu'un quart des matelots désertaient en Amérique, en dépit des peines les plus sévères dont ils étaient menacés. Suivant la coutume de ses prédécesseurs, à son arrivée à Vera-Cruz, Urdayre publia un édit qui consignait ses équipages dans leurs quartiers et interdisait aux habitants de donner refuge aux déserteurs. Dans les comptes de Pedro de Mesa, nous trouvons diverses sommes qui furent dépensées pour la capture de plusieurs des fugitifs. ${ }^{37}$

Pendant l'absence de Vallebrera, un officier nommé Juan de Ybarra fut capitaine intérimaire du Saint Julian. Comme

37 A.G.I., Contratación 3966. 
l'état du navire laissait fort à désirer, des travaux coûteux furent entrepris pour refaire la coque. Diego Vásquez, un architecte naval établi à Vera-Cruz, dirigea le carénage. ${ }^{38}$

Examinons maintenant le récit que l'auteur du Brief Discours fait de son voyage à Mexico. "Faisant cette traverse à Meschique, j'admirois les belles foretz que l'on rencontre, remplis des plus beaux arbres que l'on sçauroit souhaiter, ... la quantité que l'on veoit dans les foretz d'oiseaux de divers plumages, ... de grandes campaignes unies à perdres veue, chargez d'infinis trouppeaux de bestial...» Et c'est tout. La montée de la côte vers la meseta, les villes, les monuments aztèques, l'aspect grandiose des sommets couverts de neiges éternelles du Popocatepetl et de l'Ixtaccihnatt, - plus hauts que n'importe quelle montagne d'Europe - paraissent l'avoir laissé indifférent car il n'en dit rien.

Sa description de Mexico est également vague. «Tous les contentementz que j'avois euez à la vue de choses sy agreables n'estoient que peu de chose au regard de celuy que je receuz lors que je vis ceste belle ville de Mechique, que je ne croiois sy superbement bastye de beaux temples, pallais et belles maisons, et les rues fort bien compassées, où l'on veroit de belles et grandes boutiques de marchandz, plainnes de toutes sortes de marchandises tres riches...» C'est une description qui pourrait s'appliquer à n'importe quelle ville.

Champlain est beaucoup plus précis quand il parle du lac de Mexico, "dans lequel ilz tombent quatre grandes rivieres qui sont fort avant dans la terre, et portent batteaux: l'une s'appelle rivière de Terre Ferme, une autre rivière de Chille, l'autre rivière de Caiou, et la quatriesme rivière de Mechique ». D'où a-t-il tiré une telle description, Dieu seul le sait. Ou bien c'est le produit de son imagination, ou bien ses informateurs se sont moqués de lui.

Nous devons donc rejeter comme une invention son voyage à la capitale. Sa description du pays ne correspond d'ailleurs qu'à la luxuriante région côtière, dont la faune et la flore semblent 
l'avoir impressionné puisqu'il introduit dans son récit une embryonnaire «Histoire Naturelle des Indes » suivant la coutume de son époque. Son information est quelque peu confuse. Il croit que la cochenille est une plante. Il décrit un oiseau bizarre, le «pacho del cielo», ou oiseau du paradis (quezal), qui n'a pas de pattes, et la femelle pond ses œufs sur le dos du mâle. Cette légende a été d'ailleurs fort répandue en Europe jusqu'au $18^{\mathrm{e}}$ siècle. Comme l'oiseau servait à orner les chapeaux, on lui enlevait ses pattes avant de l'expédier en Europe, ce qui fait que beaucoup de gens croyaient qu'il n'en avait pas. Le Père Murillo Velarde a répété cette histoire fantastique dans sa Geografia Historica, publiée à Madrid en $\mathbf{1 7 5 2}$, et le naturaliste suédois Linné a dénommé l'oiseau en question « Paradisea apoda 》.

Un autre animal étrange décrit par Champlain est le dragon: « Il y a aussi des dragons d'etrange figure ayantz la teste approchante de celle d'un aigle, les ailes comme une chauve-souris, le corps comme un lezard, et n'a que deux pieds asses gros, la queue assez escailleuse, et est gros comme ung mouton. Ils ne sont pas dangereux, et ne font mal à personne, combien qu'a les voir l'on diroit le contraire. » Le dessin de cet animal qui se trouve dans le Brief Discours, le fait ressembler beaucoup plus à l'un de ces monstres qui figurent sur les monuments de l'antique Chaldée qu'à un habitant de la silve mexicaine.

Les biographes de Champlain ont déjà fait remarquer que le fondateur de Québec était assez crédule. Au Canada, il semble avoir cru à l'existence d'un monstre appelé gougon, qui était, aux dires de certains indiens, "fort effroyable et d'une telle grandeur qu'ils me disaient que le but des mats de nostre vaisseau ne luy fust pas venu jusques à la ceinture, tant ils le peignent grand, et que souvent il a dévoré et dévore encore beaucoup de sauvages ... ${ }^{39}$ Mais après tout sommes-nous qualifiés pour blâmer sa crédulité et lui jeter la première pierre ? En notre âge de scepticisme et de recherche scientifique, n'avons-nous pas les soucoupes volantes et l'abominable homme des neiges?

39 Voir aussi, Bishop, op. cit., 54; Hakluyt, op. cit., XCVIII. 
Reste à examiner le voyage de Porto-Belo, que l'auteur affirme avoir fait à son retour de Mexico. A première vue, rien de plus vraisemblable, puisque Coloma se trouvait alors à Porto-Belo, et il est fort possible que Urdayre ait voulu informer son chef de ce qui se passait en Nouvelle-Espagne. Cependant, ni les comptes de Pedro de Mesa, payeur de l'armada à Vera-Cruz, ni ceux de Tomas de Cardona, qui remplissait les mêmes fonctions à PortoBelo, ne révèlent l'envoi par l'amiral d'une seule patache ou d'un seul courrier à Porto-Belo. Mais supposons quand même que Champlain ait fait ce voyage, pendant que l'escadre de Urdayre se trouvait à Vera-Cruz. S'il a mis trois semaines pour arriver à Porto-Belo, et trois autres pour en revenir et s'il est resté, comme il le prétend, un mois à Porto-Belo, nous obtenons un total supérieur au temps qu'Urdayre a passé à Vera-Cruz, qui est de soixante jours. S'il avait été à Porto-Belo, Champlain n'aurait pas pu revenir à San Juan de Ulúa; il lui aurait fallu se rendre directement de Porto-Belo avec Coloma à La Havane où les deux escadres avaient rendez-vous pour la fin de juillet. Mais l'auteur du Brief Discours affirme non seulement qu'il est retourné à Vera-Cruz, mais aussi qu'il y a attendu quinze jours le départ de l'escadre pour La Havane: «Je m'en revins à St. Jean de Luz, où nous seiournasmes quinze jours, en attendant que l'on fist donner carenne à nos vaisseaux, pour aller à La Havanne, au rendez-vous des armées et flottes. »

Sa description de Porto-Belo est assez vague, bien qu'il mentionne les deux forts construits par Antonelli à l'entrée du port. Il commet une erreur difficile à expliquer, s'il est vrai qu'il soit resté un mois dans cette ville, quand il écrit que le Rio Chagres se jette dans l'Atlantique à Porto-Belo même, alors qu'en réalité l'embouchure de ce fleuve se trouve à une cinquantaine de kilomètres vers le sud-ouest, près de l'emplacement actuel de la ville de Colón.

Un épisode dramatique, qui ne figure pas dans le Brief Discours eut lieu à Vera-Cruz pendant le séjour de l'escadre. Ce fut l'exécution de Francisco Sequera, passager clandestin du Saint Julian, condamné à mort par Urdayre pour sodomie. On l'étrangla d'abord puis on brûla son corps sur un îlot au centre 
de la baie et en présence de toute la flotte. Le nègre qui servit de bourreau reçut 66 réaux. ${ }^{40}$

Les relations de Urdayre avec les autorités de Vera-Cruz ne semblent pas avoir été des plus cordiales. A son arrivée à San Juan de Ulúa, il leur avait interdit toute inspection de son escadre. Il prétendait que ses vaisseaux ne pouvaient contenir aucune contrebande, puisqu'ils appartenaient à une armada et non à une flotte marchande. Mais les officiers royaux de Vera-Cruz n'étaient pas de son avis et prétendaient même que l'amiral transportait sur ses navires un grand nombre de passagers clandestins, y compris des prêtres et des moines. Dans un rapport adressé au gouvernement espagnol le 20 juin, ils résumaient leurs plaintes contre l'amiral. ${ }^{41}$

Dans ce même rapport, ils annonçaient aussi l'arrivée à Vera-Cruz du dernier chargement de métaux précieux envoyé de Mexico. On s'attendait à ce que l'escadre mît à la voile le lendemain; mais le mauvais temps retarda le départ jusqu'au 29 juin $1599 .^{42}$

\section{De Vera-Cruz à SAN-LuCAR}

Après les vagues descriptions de Mexico et de Porto-Belo, nous revenons au sens de la réalité avec le récit que fait Champlain de la traversée de Vera-Cruz à La Havane: "Comme nous feusmes vingt lieus en mers, ung houracan nous prist de telle furye d'un vent de nord, que nous nous pensasmes tous perdre, et feusmes tellement escartez les ungs des autres, que nous ne peusmes rallier que à La Havanne. D'autre part nostre vaisseau faisoit telle quantité d'eau, que nous ne pensions pas éviter ce peril, car sy nous avions une demye heure de repos sans tirer l'eau, il falloit travailler deux heures sans relache, et sans la rencontre que nous fismes d'une patache, qui nous remist à nostre route, nous allions nous perdre à la coste de Campêche ... »

Les sources espagnoles ne font pas mention de cet ouragan, mais nous avons la preuve documentaire que le Saint Julian

40 A.G.I., Contratación 64 et 2966.

41 Paco y Troncoso, Epistolario de Nueva España, XIII, num. 780.

42 Urdayre, 21 février 1600. A.G.I., Indiferente General 1116. 
faisait eau et perdit contact avec l'escadre, sinon sur la côte de Campêche, tout au moins au large de la Floride. Selon Francisco Coloma, le Saint Julian était un vieux bateau qui faisait eau de toutes parts depuis le début du voyage: «La hourque Saint Julian était antique et était restée des années à sec dans un port de France, comme hors d'usage. Quand elle mit à la mer, elle fit eau de telle manière que je fus sur le point cent fois de la faire évacuer et de l'abandonner. Après de grandes difficultés elle arriva à Porto-Rico, où on la répara comme on put, et elle fut ensuite à la Nouvelle-Espagne, avec l'escadre que j'y envoyais pour les métaux précieux, et là on lui fit un grand carénage qui coûta plus de trois fois ce qu'elle valait; mais tout cela fut en vain, car elle fit eau bien davantage, et les choses arrivèrent a un tel point que l'amiral Joanes de Urdayre, commandant de l'escadre, la laissa comme perdue dans la Sonde des Tortugas. " ${ }^{43}$ Les Tortugas sont un dangereux groupe de récifs coralins à quelque distance de la côte sud de la Floride. L'escadre les traversa le 18 juillet, puisque ce jour-là l'écrivain-chef de la flotte prit acte de la latitude indiquée par chaque pilote à l'exception du pilote du Saint Julian dont il n'est fait nulle mention, preuve que le navire s'était séparé du reste de l'escadre et était probablement considéré comme perdu. ${ }^{44}$

Malgré cela, selon le Brief Discours, le Saint Julian arriva à La Havane avant Urdayre, ce qui n'est pas après tout impossible: "Arrivames à la Havanne et nous y trouvasmes nostre general, mais nostre admirante n'y estoit pas encores arrivé, qui nous faisoit croire qu'il estoit perdu; toutefoys il se rendit bien tost après avec le reste de ses vaisseaux. »

Champlain est d'ailleurs d'accord avec les sources espagnoles quand il affirme que Urdayre arriva après Coloma. Ce dernier était parti de Carthagène le 3 juillet et était rentré à La Havane le 27, tandis que l'amiral n'y arriva que le $1^{\text {er }}$ août.

Peu après, le Saint Julian fut désarmé et vendu aux enchères par ordre de Coloma. ${ }^{45}$ Nous ignorons la date exacte de cette

43 Simancas, Estado K 1603, B 86, num. 115.

14 A.G.I., Contratación 64 (parage des Tortugas, 18 juillet 1599).

45 Voir note relative au Saint Julian: * cette hourque s'échoua et se vendit à La Havane parce qu'elle ne pouvait plus naviguer à cause de ses voies d'eau, en ... a oût 1599 ஓ. A.G.I., Contratación 2956. 
vente, mais elle dut avoir lieu dans la première quinzaine d'août, car le 15 de ce mois le capitaine du navire, Yeronimo de Vallebrera, fut promu alferez real. ${ }^{46}$ Sebastian Rodriguez toucha sa paie de guardian (quartier-maître) du Saint Julian jusqu'au 12 août, et commença à servir le jour suivant comme simple marin à bord de la patache Sandoval. Simon de Juan, contremaître du Saint Julian, fut transféré au galion San Augustin.47 Le bateau français capturé par Urdayre, sur la côte de St-Domingue, fut aussi vendu à La Havane pour 1300 ducats. Nulle part dans le Brief Discours trouvons-nous mention de la vente du Saint Julian.

A cette époque, se trouvaient réunis à La Havane plus de 70 vaisseaux avec 4,000 hommes à bord, pour retourner en Espagne, car Coloma avait ramené avec lui de Carthagène et de PortoBelo la flotte de Terre Ferme, commandée par Sancho Pardo Osorio, et la capitane de Luis Fajardo, général de l'armada de 1598. Cette armada était revenue en Espagne en mars 1599, à l'exception de la capitane, le galion Santo Domingo, qui avait perdu ses mâts dans un ouragan et avait dû regagner Carthagène, où Fajardo se trouvait encore à l'arrivée de Coloma. Quand les trois généraux ancrèrent à La Havane le 27 juillet, le gouverneur leur transmit des instructions fraîchement reçues d'Espagne, qui donnaient le commandement suprême des flottes réunies à $\mathrm{Fa}$ jardo, tout en maintenant Coloma à la tête de ses galions. Il en résultait une situation assez délicate, puisque cinq ans plus tôt Fajardo avait été sous les ordres de Coloma et n'avait pu s'entendre avec lui. Les plaintes que Fajardo avait portées contre son chef avaient été en grande partie la cause de la révocation de ce dernier par le Conseil des Indes. Fajardo avait même menacé de démissionner si on l'obligeait de nouveau à servir sous Coloma. En 1599 la situation se trouvait renversée, puisque Coloma se trouvait subordonné à Fajardo. Mais à la surprise générale les deux généraux s'entendirent fort bien et paraissent même être devenus bons amis. Fajardo ne prit d'ailleurs aucune décision importante sans avoir auparavant consulté son collègue.

Nous avons déjà vu que Coloma avait été révoqué pour trois ans en 1595 à cause des nombreuses exactions qu'il avait com-

46 A.G.I., Indiferente General 2680.

47 A.G.I., Contratación 2958. 
mises. Voulut-il se racheter quatre ans plus tard et donner alors une preuve éclatante de sa probité ? Toujours est-il qu'à La Havane il fit mettre aux fers la majorité des maîtres de son armada, accusés de malversations diverses, et les ramena prisonniers en Espagne. Parmi eux se trouvait Bartolomé López, maître du Saint Julian. Ce dernier était compromis dans la vente, à Porto-Rico et en Nouvelle-Espagne, du vin et de l'huile que Zubiaur avait fait charger illégalement sur le navire à SanLucar. ${ }^{48}$

Après l'arrivée de Urdayre à La Havane le $1^{\text {er }}$ août, il ne restait plus qu'à faire l'aiguade et à prendre le chemin du retour. Les flottes réunies étaient sur le point de partir, quand on apprit qu'une escadre hollandaise de 80 navires s'était emparée de la Grande Canarie, et on se mit à craindre que les vaisseaux rebelles, embusqués dans les eaux de l'archipel, n'attaquent à leur retour d'Amérique les galions espagnols, pour leur enlever leur riche cargaison d'or, d'argent, de perles, pierres précieuses, cochenille et indigo, évaluée à plus de 12,000,000 de ducats. Le 7 août, se réunit un conseil des généraux, amiraux et pilotes, présidé par Antonio González, membre du Conseil des Indes, qui revenait d'une tournée d'inspection en Amérique du Sud. On décida d'attendre que le danger soit passé. Mais on sut bientôt que l'occupation de la Grande Canarie par les Hollandais n'avait duré que huit jours, et que la flotte ennemie se dirigeait vers le détroit de Magellan. Il n'y avait donc plus de danger, et l'on pouvait entreprendre en toute sécurité le voyage de retour; cependant les flottes restèrent à La Havane parce que des instructions reçues d'Espagne interdirent le départ jusqu'à nouvel ordre. L'autorisation de se mettre en route arriva enfin le 23 décembre; mais le mauvais temps retarda le départ jusqu'au 6 janvier $1600 .{ }^{49}$

Le séjour à La Havane dura donc cinq mois (quatre selon Champlain, qui ne donne aucune explication de ce délai). C'est au cours de cette longue attente que l'auteur du Brief Discours place son voyage à Carthagène, où il affirme avoir séjourné un

48 A.G.I., Escribania de Camara, 1012 A.

49 Fajardo, 24 février 1600 . Coloma, 23 février 1600. Urdayre, 21 février 1600. A.G.I. Indiferente General 1116. 
mois et demi et il ajoute: «Partant dudict lieu de Cartagène, je m'en retournay à La Havanne trouver nostre general, qui me fist fort bonne réception, pour avoir veu par son commandement les lieux où j'avois esté. » De nouveau il cherche à se donner de l'importance. A-t-il vraiment été à Carthagène ?

Parmi les instructions que les généraux avaient reçues à leur arrivée à La Havane se trouvait un ordre interdisant l'envoi d'avisos ou de courriers, de peur que leur capture ne révélât à l'ennemi les mouvements des flottes espagnoles. Certes, il est possible que cet ordre ne s'appliquât qu'aux messages pour l'Espagne. Cependant, dans les comptes des trois flottes réunies à La Havane (celles de Fajardo, Coloma, et Sancho Pardo), nulle mention n'est faite de l'envoi d'un aviso à Carthagène, ou à aucun autre port durant les cinq mois d'attente à La Havane. Les flottes avaient d'ailleurs l'ordre de se tenir prêtes à partir d'un jour à l'autre. En allant à Carthagène, Champlain risquait fort de revenir à La Havane après leur départ et d'avoir à attendre un an avant de pouvoir rentrer en Espagne.

Quant à sa description de Carthagène, il aura pu l'obtenir de n'importe quel marin qui y aura séjourné: "Ce lieu est ung tres bon port, où il y a belle entrée, à l'abry de tous ventz, fors du nord norouest, qui frappe dans ledict port, dans lequel il y a trois isles. Le Roy d'Espaigne y entretient deux galleres. » De la traversée entre La Havane et Carthagène il ne donne aucun détail, pas plus qu'il n'en avait donné de sa traversée précédente de Vera-Cruz à Porto-Belo.

Nous avons vu que l'ordre de départ ne fut reçu à La Havane que le 23 décembre. Fajardo, Coloma et Urdayre devaient rentrer immédiatement avec les galions; mais les vaisseaux marchands devaient attendre jusqu'au 15 mars pour mettre à la voile, sous le commandement de Sancho Pardo. ${ }^{50}$ L'auteur du Brief Discours ne pouvait plus voyager à bord du Saint Julian, puisque ce navire avait été vendu. Est-il revenu sur un des galions, ou a-t-il attendu à La Havane le départ de Sancho Pardo ?

${ }^{50}$ Lettres du Conseil des Indes à Fajardo, Coloma et Sancho Pardo (Octobre 1599). A.G.I. Indiferente General 746. 
Sans le dire textuellement, il nous donne à entendre qu'il rentra avec les galions, puisqu'il relate un épisode mentionné aussi par les sources espagnoles: la capture de deux bateaux anglais au large du Cap Saint-Vincent, par Fajardo et Coloma, qui réclamèrent plus tard leur part du butin (le cinquième généralement attribué aux généraux) ${ }^{51}$

De la traversée même, Champlain ne dit pas grand'chose. Il raconte cependant qu'ils naviguèrent vers le nord par le canal de Bahama, entre Cuba et la Floride, jusqu'à passer en vue de l'île Bermude, et il ajoute: "Il faut que je dye encor qu'à costé dudict canal de Bahan, au sudouest, l'on voit l'isle St. Domingue, dont j'ay parlé cy dessus ... Encore que j'aye cy dessus représenté la dicte isle de St. Domingye, je figureray neanmoins ici la coste d'icelle vers le canal de Bahan. » Pas même avec un télescope il n'aurait pu apercevoir St-Domingue du canal de Bahama, puisque la distance est de plus de sept degrés. Aura-t-il confondu Andros avec St-Domingue?

Francisco Coloma et ses galions arrivèrent à San-Lucar de Barrameda le 26 février 1600, treize mois après avoir quitté l'Espagne. ${ }^{52}$ L'auteur du Brief Discours prétend qu'il fallut exactement le double pour effectuer le voyage: «...l'achèvement de nostre voiage, auquel je demeuroy depuis nostre partement de Siville, tant sur mer que sur terre, deux ans deux mois ».

\section{CONCLUSION}

Quelles conclusions pouvons-nous tirer de notre confrontation du Brief Discours avec les sources? Quelle part devonsnous faire à la vérité, à l'erreur, et à la fantaisie ?

Tout d'abord, nous devons rejeter comme incompatible avec l'itinéraire suivi par l'armada de Coloma, et plus particulièrement par l'escadre de Joanes de Urdayre, tout ce qui se rapporte à la Guadeloupe, la Marguerite et les Caymans. Nous devons aussi faire les plus grandes réserves sur les prétendus voyages à

51 Fajardo 1601. A.G.I., Indiferente General 2680.

52 Agustin de la Guerra, 18 avril 1600. A.G.I., Contratación 3332. 
Mexico, Porto-Belo et Carthagène, bien que l'auteur affirme les avoir faits par ordre ou avec l'autorisation de ses supérieurs.

Quant au reste, devons-nous conclure qu'il est vraiment parti pour les Indes Occidentales avec l'armada de Francisco Coloma ? Quelques parties de son récit semblent avoir été vécues, telles que la croisière le long de la côte nord de St-Domingue (bien qu'il se trompe sur l'emplacement de Monte Christi), l'escarmouche de Gonaives, et la traversée de Vera-Cruz à La Havane, quand le Saint Julian faillit se perdre. En revanche, la description de Porto-Rico, à l'arrivée de l'armada, nous fait rêver. Plus extraordinaire encore est le fait que l'auteur ne souffle mot de la vente du Saint Julian et nous donne l'impression qu'il a effectué tout le voyage sur le même navire. Nous avons déjà remarqué que, depuis le départ de San-Lucar, nous ne trouvons dans le Brief Discours le nom d'un seul bateau, ni d'une seule personne, ni une seule date précise.

Il est fort possible que Champlain n'ait pas fait le voyage. Peut-être sa condition d'étranger et le fait qu'il n'était pas marin de profession l'ont-ils empêché de s'engager dans l'armada, et il aura pu rester tout le temps à San-Lucar, où résidait une importante colonie française composée surtout de Bretons. Depuis le temps où la Bonne Duchesse Anne avait été fiancée au fils des Souverains Catholiques, San-Lucar était fréquenté par de nombreux marins et marchands bretons, qui y avaient leur consulat et leur faubourg tout près du fleuve, à l'endroit où se trouve aujourd'hui la «Calle de los Bretones ». Il a pu attendre là, ou à Cadix, ou peut-être est-il parti avec Guillermo Eleno pour servir dans l'escadre de Zubiaur. Au retour des galions de Coloma, il aura pu interroger quelques-uns des marins français qui avaient fait partie de l'équipage du Saint Julian, et d'après les renseignements ainsi recueillis, il aura rédigé le Brief Discours. Quant aux cartes, il est possible qu'il en ait copié quelques-unes sur des cartes de pilotes et qu'il ait inventé les autres.

Ou bien, l'auteur du Brief Discours a pu faire le voyage, mais n'aurait commencé la rédaction de son récit que beaucoup plus tard, dix ou quinze après, quand ses souvenirs commençaient à s'estomper. Les épisodes dont il se souvient le mieux et qu'il 
décrit avec le plus de vie, sont aussi les plus dramatiques: la bataille de Gonaives, et la traversée de Vera-Cruz à Cuba faite au péril de sa vie. Mais, qu'il ait effectué le voyage ou non, il y a inclus Porto-Belo et Carthagène parce que Coloma y a été. S'il mentionne la Marguerite, c'est parce que chaque armada y envoyait une patache, et sa description de la Guadeloupe se doit au fait que les flottes se rendant en Amérique avaient l'habitude d'y faire l'aiguade. Dans sa narration il a compris tous les lieux visités par une partie ou la totalité de l'armada qui se rendait annuellement aux Indes Occidentales, sans tenir compte de l'itinéraire vraiment suivi par le Saint Julian. Il a pu apprendre ce qu'il sait des Caymans de quelque corsaire français. Il reproduit aussi dans son récit des descriptions fantastiques de specimens de la faune mexicaine, faisant ainsi preuve d'une grande crédulité.

Mais si nous acceptons cette seconde alternative, à savoir que le Brief Discours a pu être rédigé plus de dix ans après le retour des galions de Coloma, par un homme dont la mémoire était quelque peu confuse, nous ne pouvons en attribuer la paternité à Samuel Champlain. Ce dernier aurait eu des motifs pour l'écrire quand il était encore en Espagne, ou fraîchement revenu en France, afin de se faire un nom, attirer sur lui l'attention des autorités et obtenir quelque emploi ou charge. Après 1602, il était trop absorbé par ses voyages au Canada pour rédiger le Brief Discours. En le faisant d'ailleurs à ce moment, il aurait pu compromettre sa réputation. Autant que je le sache, le Brief Discours est la seule œuvre attribuée à Champlain qui soit restée manuscrite jusqu'à sa mort. Pourquoi ne l'a-t-il pas comprise dans l'édition générale de ses œuvres ? Avait-il peur d'être traité de menteur, ou n'en était-il pas l'auteur?

Le manuscrit original manque malheureusement, ce qui empêche toute expertise de l'écriture. Le nom de Champlain ne figure pas dans le texte du «Brief Discours》, mais seulement dans le titre et de la façon suivante: Brief Discours des choses les plus remarquables que Samuel Champlain de Brouage a reconnues aux Indes Occidentales. Après tout le titre a pu être ajouté plus tard et ne constitue pas en soi une preuve de paternité. $\mathrm{Y}$ 
a-t-il dans les documents de l'époque un indice qui nous permette d'établir un rapport entre Champlain et le Brief Discours?

Pendant des mois j'ai fouillé dans diverses sections des Archives des Indes à Séville sans pouvoir rencontrer une seule fois le nom du fondateur de Québec. J'aurais peut-être eu plus de chance à l'Archivo del Mar Oceano à San-Lucar de Barrameda et j'aurais même pu y trouver la liste complète des marins du Saint Julian à l'époque, où le navire arriva en Espagne, car il existe un document de la Maison de la Contratación, daté du 23 décembre 1598, qui contient le paragraphe suivant: "Nous ne pouvons ajuster les comptes de ce qui se doit aux équipages du général $\mathrm{P}$. de Zubiaur, parce que les listes originales sont à l'Armada del Mar Oceano ». ${ }^{53}$ Malheureusement, l'Archivo del Mar Oceano n'est pas ouvert au public. Son propriétaire, le duc de Medina Sidonia, en interdit formellement l'entrée. Son Excellence, qui a eu la bonté de me recevoir après trois mois d'attente, a bien voulu m'expliquer qu'il avait peur d'être volé.

D'autre part, il semble que Champlain était à Cadix en 1601. 24 ans plus tard, le 29 décembre 1625, il céda à un ami, Charles Lebert, $\mathrm{Sr}$ du Carlot, de Brouage, une propriété qu'il avait reçue " au moyen de la donnation a luy faicte par deffunct Guillaume Hellaine, de nation marsellois, naturel de la citté de Marseille, estant en la cité de Cadis, par act passé audict Cadiz en Espaigne par devant Marc de Ribers, notaire publicq en ladicte citté, le 2 jour de juillet mil six cens ung ... » ${ }^{54}$

Ce transfert de propriété qui avait eu lieu à Cadix en 1601 est d'intérêt primordial, car il montre que Champlain connaissait Guillermo Eleno, capitaine du Saint Julian, puisque « Guillaume Hellaine, de nation marseillois, naturel de la citté de Marseille» et «Guillermo Eleno Provençal » sont évidemment la même personne. Malheureusement le texte original du document rédigé à Cadix le 2 juillet 1601 par le notaire Marcos de Ribera n'existe plus. J'ai fait tout mon possible pour le retrouver; mais les registres de Marcos de Ribera pour l'année 1601 ne se rencontrent pas

53 A.G.I. Contratación 5112. $80 \mathrm{ss}$.

54 Publié par A. Leo Leymarie dans Nova Francia (octobre 1925), I : 
à l'Archivo de Protocolos de Cadix. Il semble qu'ils se sont perdus lors du transfert de l'archivo à son site actuel, il y a six ans. Pendant leur transport, plusieurs liasses se désintégrèrent et furent jetées au rebut.

Donc, il semple que Champlain et le Capitaine Provençal se trouvèrent ensemble à Cadix. Etaient-ils vraiment parents? Si Champlain était le neveu de Hellaine, il n'y fait aucune allusion dans le document de 1601. En outre, apparemment induit en erreur par le fait que le capitaine du Saint Julian était surnommé « Provençal », Champlain prétend qu'il était « de nation marseillois, naturel de la citté de Marseille». Mais Hellaine n'était point de Provence et encore moins naturel de Marseille, puisqu'il naquit à Brouage, tout comme Champlain, ainsi qu'il l'affirme lui-même dans une déclaration jurée faite à Fumchal (Madère) le 3 août 1600 .

A cette époque, Eleno commandait la capitane de l'escadre du Détroit de Gibraltar, dont le général était Pedro de Zubiaur. Ce dernier, pendant l'été de 1600 , conduisit sa flotte à Madère. Comme il approchait de la côte, il aperçut trois navires suspects qui mettaient à la voile comme s'ils essayaient de fuir. Zubiaur les rejoignit, les intercepta, et les obligea à revenir au port, prétextant que deux d'entre eux étaient anglais et le troisième hollandais, donc ennemis de l'Espagne. Mais les capitaines des trois vaisseaux capturés ne tardèrent pas à protester, les deux premiers affirmant qu'ils n'étaient point sujets de la Reine Vierge, mais du roi Jacques d'Ecosse, ami du Roi Catholique; quant au troisième, il déclarait qu'il n'était pas hollandais mais flamand, et par conséquent sujet du roi d'Espagne. Il devait être fort difficile de distinguer un Écossais d'un Anglais, un Flamand d'un Hollandais, et un catholique d'un huguenot, et l'ex-corsaire Zubiaur n'était pas homme à se préoccuper beaucoup de ces nuances.

L'évêque de Funchal, qui était gouverneur de l'île, ordonna une enquête pour établir la nationalité des trois vaisseaux. Le 3 août 1600 , les témoins cités par Zubiaur déposèrent en sa faveur. Deux d'entre eux étaient français: Jean Carneau, natif 
de Roscoff et pilote de la capitane, et Guillermo Eleno, capitaine du même navire, qui déclara être âgé de «soixante ans plus ou moins ». Le témoignage de ce dernier est précédé de la note suivante du juge d'instruction: "Aujourd'hui, je prends et reçois serment selon la loi du capitaine Guillermo Eleno Provençal, français, natif de Brouage ». A la fin de la déposition apparaissent trois signatures: celle du juge, celle de Zubiaur, et celle de Guileume Eleno..$^{55}$

Le problème qui se pose maintenant est le suivant: si Champlain était vraiment le neveu du Capitaine Provençal, s'il le connaissait aussi intimement que le laisse entendre l'auteur du Brief Discours, comment ignorait-il que son oncle était comme lui natif de Brouage? Comment pouvait-il le prendre pour un Provençal? Ou est-ce qu'en 1625 il se rappelait fort mal certains de ses proches parents? Il est vrai que Guillaume Hellaine était probablement décédé depuis longtemps. En 1600, il était âgé de 60 ans. Certains documents des Archives de Simancas nous portent même à croire qu'il mourut peu de temps après.

Nous savons en effet qu'au début de 1601, il avait perdu son commandement et figurait comme simple marin sur la liste de l'équipage du galion Crucifixo de Lezo. En avril il était malade et se trouvait incapable de servir. A deux reprises, des acomptes sur sa solde lui furent versés par ordre de l'Adelantado Mayor de Castille, commandant en chef de l'Armada del Mar Oceano, «parce qu'il est malade et dans le besoin ».56 L'acte passé à Cadix le 2 juillet 1601 par devant le notaire Marcos de Ribera était peut-être son testament, et il est possible qu'il soit mort peu après.

Quelle nécessité avait poussé le capitaine Provençal à servir ainsi à son âge comme simple matelot? En 1600, le Conseil de Guerre l'avait proposé pour un entretenimiento (pension) de 20 ducats par mois - presque le double de la solde ordinaire d'un

55 Simancas, Guerra Antigua 572.

56 Voir les comptes de Juan Pascual, payeur de l'Armada del Mar Océano (1597-1601) aux Archives de Simancas, Contaduria Mayor (2da. Epoca, liasse 406, fol. 115 et 119. 
capitaine. ${ }^{57}$ Peut-être qu'il n'avait pas encore obtenu sa pension, ou qu'il n'avait pas commencé à la toucher. A cette époque, les troupes et les équipages espagnols restaient parfois deux ans sans être payés.

Mais revenons à Champlain. Nous trouvons dans son œuvre intitulée Les Voyages de la Nouvelle France Occidentale, dicte Canada, un passage où il affirme avoir été aux Indes Occidentales et qui est rédigé en ces termes: "Sur ces entrefaites je me trouvay en cour, venu fraischement des Indes Occidentales, ou j'avais esté près de deux ans et demy, après que les Espagnols furent partis de Blavet, et la paix faite en France, où pendant les guerres j'avois servy Sadite Majesté sous Messeigneurs le marechal d'Aumont, de Sainct Luc, et marechal de Brissac ... $\gg^{58}$

Il ne fait nulle mention de l'armada de Coloma. A-t-il effectué le voyage d'autre manière, peut-être sur un bateau corsaire? Nous n'avons aucune preuve documentaire qu'il ait servi dans les guerres de la Ligue sous D'Aumont, St. Luc et Brissac, mais cela est après tout fort possible, car ces trois généraux commandèrent successivement l'armée française en Bretagne. Dès décembre 1594, D'Aumont avança jusqu'à douze lieues de Vannes, et le gouverneur de la ville, René d'Aradon, beau-frère de La Hautière, se sentit si menacé qu'il appela à son secours Jean del Aguila, gouverneur espagnol de Blavet.59

L'auteur du Brief Discours affirme également avoir servi en Bretagne sous ces trois généraux, en termes presque identiques, et tout au début de son récit, ainsi qu'il suit: «Aiant esté emploié en l'armée du Roy qui estoit en Bretaigne soubs messieurs le marechal d'Aumont de St. Luc et Marechal de Brissac ... » La ressemblance est vraiment impressionnante. Devons-nous y voir une preuve de paternité commune, ou celle d'un plagiat? Les deux hypothèses sont également vraisemblables.

Si nous comparons la phrase mentionnant les trois généraux dans les deux textes, nous remarquons dans le Brief Discours

57 Simancas. Guerra Antigua 567.

58 Edition de 1632, p. 39.

59 Juan del Aguila au Roi, 17 décembre 1594. Simancas Estado K. 1591, B 80, num. 161. 
une erreur de ponctuation, insignifiante à première vue, mais qui a une certaine importance: la virgule a disparu entre d'Aumont et de St. Luc, ce qui fait croire que les deux noms sont ceux d'une seule personne. Ce manque de virgule paraît avoir dérouté le copiste de Bologne, puisqu'il a changé «messieurs » en «monsieur ». De même les éditeurs de la Hakluyt Society semblent avoir cru que D'Aumont et St. Luc étaient la même personne, bien que le manuscrit qu'ils ont utilisé - celui de Providence ait conservé «messieurs ». Puisque la virgule manquait dans les deux copies de Bologne et de Providence, elle a dû faire défaut dans le manuscrit original.

Si nous acceptons l'hypothèse d'un plagiat, nous pouvons établir le raisonnement suivant. L'auteur du Brief Discours avait un manuscrit illustré dont il voulait tirer parti, et il aura décidé de mettre à profit la renommée de Samuel Champlain. Après la publication d'une des premières éditions des Voyages de la Nouvelle France Occidentale, il aura plagié le passage relatif à la part que Champlain avait prise aux guerres de la Ligue et à son voyage aux Indes Occidentales, l'utilisant comme introduction de son propre ouvrage, et intitulant son manuscrit de manière à faire croire que Champlain en était l'auteur. Il aura dû aussi doubler le temps que l'armada de Coloma avait mis à faire le voyage, et changer les treize mois qu'il avait fallu en réalité en deux ans et deux mois, afin d'harmoniser son récit avec l'affirmation de Champlain qu'il avait été aux Indes Occidentales « près de deux ans et demy ».

Il est donc possible que Champlain ne soit pas l'auteur du Brief Discours, mais cette possibilité ne repose sur aucune preuve concrète, car la concordance des deux passages cités plus haut, et le fait que le Capitaine Provençal et Champlain se connaissaient, pourraient faire croire que le fondateur de Québec est l'auteur du Brief Discours. M. Delafosse, archiviste de la CharenteMaritime, qui prépare une étude sur le Capitaine Provençal, m'écrit que ce dernier fut dans la deuxième moitié du $16^{\mathrm{e}}$ siècle un corsaire réputé et que sa carrière aventureuse le conduisit au Brésil. Provençal a peut-être fourni à l'auteur du Brief Discours une partie des cartes et des renseignements utilisés par celui-ci. 
Champlain a-t-il navigué sur le Saint Julian? Est-il allé en Amérique avec l'armada de Francisco Coloma? A-t-il écrit le «Brief Discours»? Nous devons nous limiter à des conjectures jusqu'à ce que nous soyons plus amplement informés. Peut-être découvrirons-nous la clef du mystère si nous retrouvons le manuscrit original du Brief Discours, ou si le duc de Medina Sidonia surmonte sa peur des voleurs. Quoi qu'il en soit, respectons la mémoire de Samuel Champlain, dont la gloire mondiale bien méritée ne saurait être entamée par une possible erreur de jeunesse.

Madrid, Espagne

\section{L.-A. Vigneras}

Nous annonçons bien volontiers cette entreprise d'histoire dont M. W. L. Morton de l'Université du Manitoba a pris la direction: une Histoire du Canada depuis l'an 1000, en 16 vols et qui paraîtra de 1949 à 1967.

1. T. J. Oleson, The Early voyages and northern approches, 1000-1632.

2. Marcel Trudel, The origins of New France, 1524-1663.

3. W. J. Eccles, The Province of New France under Louis $X I V, 1663-1672$.

4. Guy Frégault, The Empire of New France, 1701-1760.

5. Hilda Neatby, Quebec, 1760-1791.

6. Fernand Ouellet, Lower Canada, 1792-1840.

7. G. M. Craig, Upper Canada, 1792-1840.

8. W. S. MacNutt, The Atlantic Provinces, 1713-1864.

9. J. M. S. Careless, The Union of the Canadas, 1841-1859.

10. E. E. Rich, The North and Fart West, 1668-1857.

11. W. L. Morton, The Federation of British North America, 1857-1873.

12. J. T. Saywell, The Testing of Confederation, 1874-1896.

13. H. B. Neatby, The Emergence of Modern Canada, 18971921.

14. F. W. Gibson, The Canadian Nation, 1922-1939.

15. M. T. Zaslow, The Conquest of the North, 1858-1960.

16. Sera annoncé plus tard: Epilogue, 1940-1967. 